\title{
Experimental Bilirubin Encephalopathy: Importance of Total Bilirubin, Protein Binding, and Blood-Brain Barrier
}

\author{
RICHARD P. WENNBERG AND A. J. HANCE \\ Division of Neonatology, Department of Pediatrics, and Department of Pharmacology, School of Medicine, \\ University of California, Davi. California 95616
}

\begin{abstract}
The cause of bilirubin encephalopathy has been variously ascribed to elevated total serum bilirubin concentration, high free bilirubin levels (or impaired albumin binding), and disruption of the blood-brain barrier. An experimental rat model for acute bilirubin encephalopathy was developed in which these three factors could be varied independently. Osmotic opening of the blood-brain barrier in the right hemisphere was produced by infusing a hypertonic arabinose solution into the right carotid artery. The total bilirubin level and bilirubin binding state were varied by adjusting the amount of bilirubin infused intravenously and/or by infusing human serum albumin. Brain electrical activity (EEG) served as an indicator of developing encephalopathy. Neither staining nor EEG changes occurred if the blood-brain barrier remained intact. Bilirubin staining without EEG evidence of encephalopathy sometimes occurred when the blood-brain barrier was open. Discriminant analysis showed that EEG changes were best predicted by the degree of blood-brain barrier opening (as indicated by brain bilirubin content) and by the quality of serum bilirubin binding. Serum total bilirubin concentration was not an important discriminator of encephalopathy. (Pediatr Res 20: 789-792, 1986)
\end{abstract}

\section{Abbreviations}

HSA, human serum albumin

BSA, bovine serum albumin

BBB, blood-brain barrier

Thirty-five years after the indictment of bilirubin as the cause of kernicterus (1), our understanding of the pathogenesis of bilirubin encephalopathy remains incomplete. Total bilirubin concentration (2), free bilirubin concentration $(3,4)$, and reduced serum bilirubin binding (5-7) have been implicated as critical elements in the pathogenesis of this condition, but fail to explain fully the variable response of infants to hyperbilirubinemia. More recently, disruption of the $\mathrm{BBB}$ has been proposed to be the cause of bilirubin toxicity (8).

Infusion of a hypertonic solution of urea or arabinose into a carotid artery of a rat will temporarily open the BBB in the ipsilateral hemisphere $(9,10)$. Large proteins such as albumin may then gain access to the interstitial space of brain. If Evans Blue dye is bound to the albumin, the ipsilateral hemisphere will be stained blue (10). If bilirubin is bound to the albumin, the brain will turn yellow, as reported by Levine et al. (8). Levine et

Received January 10, 1986; accepted April 7, 1986.

Correspondence Richard P. Wennberg, M.D., Division of Neonatology, University of California, Davis, CA 95616 al. (8) suggested that kernicterus and bilirubin toxicity are caused by the albumin-bilirubin complex crossing a disrupted BBB and argued that the level of free bilirubin or nature of bilirubin binding may not be important in the development of bilirubin encephalopathy. However, Levine et al. (8) did not provide histological, physiological, or behavioral evidence of bilirubin neurotoxicity to support this view.

There is, on the other hand, abundant in vitro evidence that albumin-bound bilirubin is not toxic (11-15) and that the concentration of free bilirubin acid may be responsible for cell injury $(4,16)$. Recently, we examined the possibility that yellow staining may not be harmful if it represents the transport of albumin bound bilirubin across a disrupted BBB. To test this hypothesis, we utilized Levine's rat model, and, in addition, cerebrocortical electrical activity was recorded as an indicator of acute bilirubin toxicity. The initial studies (17) indicated that 1) neither brain staining nor EEG changes could be produced in young adult rats with intact $\mathrm{BBB}$ who were exposed to severe hyperbilirubinemia (serum bilirubin $42-74 \mathrm{mg} / \mathrm{dl}$ ) for $60 \mathrm{~min}$; 2) following osmotic opening of the BBB, the affected hemisphere was stained yellow following bilirubin infusion; 3) yellow staining was not always associated with encephalopathy as judged by EEG changes; 4) rats infused with human serum albumin, which binds bilirubin more tightly than does rat serum, required a higher total bilirubin level before EEG changes were observed. These data strongly suggested that while osmotic opening of the BBB might facilitate the equilibrium of bilirubin between albumin and brain cells, encephalopathy would only occur when the free bilirubin is sufficiently elevated to drive toxic levels of bilirubin onto functional cell membranes. This study examines further the relationship between serum total bilirubin concentration, barrier opening, serum bilirubin binding state, and the development of encephalopathy.

\section{METHODS}

Twenty-seven young male Wistar rats, 300-350 g, were anesthetized with pentobarbital sodium $(60 \mathrm{mg} / \mathrm{kg}$ intraperitoneal). The calvarium was exposed and cleaned of periosteum. Stainless steel screw electrodes were placed through burr holes drilled anteriorally and posteriorally $3-4 \mathrm{~mm}$ on each side of the midline, with a ground electrode overlying the frontal sinus. Bipolar anterior to posterior recordings were made from the right and left electrode pairs on a Grass Model 7D Polygraph. Temperature was monitored by a rectal probe. A tracheostomy was performed to ensure a patent airway. Catheters were placed in a femoral artery for blood sampling and in a femoral vein for infusion of bilirubin and albumin. A PE10 catheter was placed in the right external carotid artery with the tip lying near the branch of the internal carotid artery for infusion of hypertonic arabinose solution. 
In order to independently vary the quality of binding and bilirubin levels, rats were primed with either $1.5 \mathrm{~g} / \mathrm{kg}$ HSA $(25 \%$ HSA, Cutter Laboratories) (12 rats) or an equivalent volume of saline (15 rats). Ten minutes later, $0.8-1.0 \mathrm{ml}$ of a saturated arabinose solution was infused into the carotid artery over $30 \mathrm{~s}$. The infusion was terminated when a decrease in amplitude in the EEG appeared, indicating opening of the barrier (Fig. 1). The volume of arabinose administered was less than that reported by Levine et al. (8) since marked opening of the BBB invariably produced a prolonged cessation of electrical activity over the ipsilateral hemisphere. Two min after the arabinose infusion or when cortical electrical activity recovered, a bilirubin solution was infused with a Harvard Pump via the femoral vein. Fifteen mg bilirubin (Sigma) was dissolved in $2.5 \mathrm{ml}$ of $0.1 \mathrm{~N} \mathrm{NaOH} /$ isotonic saline (final pH ca 9.5) and administered at a rate of $1.71 \mathrm{mg}$ bilirubin $/ \mathrm{min}(0.29 \mathrm{ml} / \mathrm{min})$. Infusion of alkaline bilirubin had a negligible influence on blood $\mathrm{pH}$. The room was darkened during the bilirubin infusion to minimize photoisomerization of the pigment. The infusion was continued for 4 to $8 \mathrm{~min}$ in order to establish a range of maximum total bilirubin concentration. The infusion was stopped immediately if EEG changes appeared in order to identify threshold conditions for bilirubin levels and binding state.

Blood was sampled immediately after termination of the bilirubin infusion for measurement of total bilirubin and assessment of serum binding. A second sample was obtained 5 min later following which $7 \mathrm{mg}$ of Evans Blue $(10 \mathrm{mg} / \mathrm{ml}$ saline) was infused through the femoral vein. Two min later the animal was sacrificed by infusion of 8 molar $\mathrm{KCl}$. A thoracotomy was performed immediately and icteric plasma and red cells were removed by perfusing the animal with $120-150 \mathrm{ml}$ isotonic saline through a catheter placed in the left ventricle. The brain was removed and a single coronal section was made at the level of

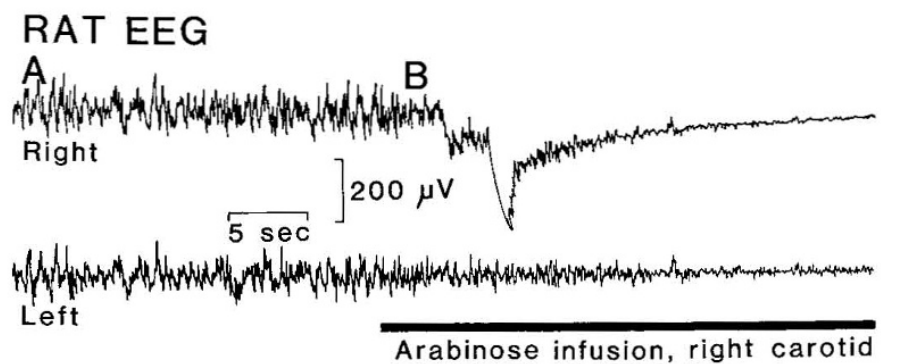

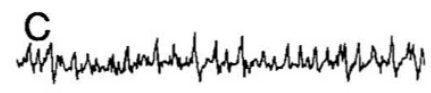

D

(severe)

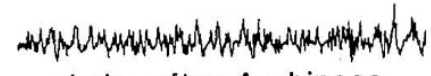

4 min. after Arabinose

Bilirubin infusion complete

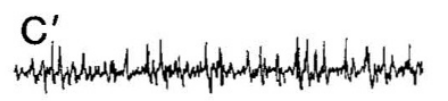

$\mathrm{D}^{\prime}$

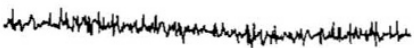

(moderate)
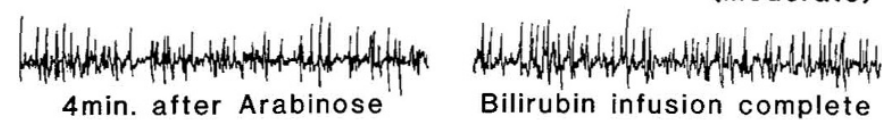

Fig. 1. Typical electroencephalographic changes. $(A-D)$ EEG changes in a single rat before $(A)$, during $(B)$, and 4 min following $(C)$ infusion of saturated arabinose solution in the right carotid artery. $(D)$ Subsequent severe changes in EEG following bilirubin infusion. $\left(C^{\prime}-D^{\prime}\right)$ EEG recording in a rat demonstrating moderate changes induced by bilirubin. the anterior thalamus to examine staining of deep structures. The brain was then divided into brainstem/cerebellum, right and left hemispheres, and frozen at $-10^{\circ} \mathrm{C}$ until analyzed.

After weighing each brain segment and homogenizing in $2 \mathrm{~N}$ acetic acid, the bilirubin was extracted with chloroform/methanol and partitioned into the chloroform phase by adding water (18). The bilirubin concentration was determined by measuring the absorptivity at $452 \mathrm{~nm}$. Two extractions were performed with each brain, and the total brain bilirubin extracted was expressed in $\mu \mathrm{g}$ bilirubin/g wet weight. Bilirubin content of brain was presumed to primarily represent interstitial bilirubin-albumin and, therefore, reflect the degree of BBB disruption (8).

In a preliminary study (17) serum binding was measured using the peroxidase method. Results were believed to be unreliable due to the high intrinsic oxidation rate in many sera and because human and rat albumin have different albumin-bilirubin dissociation rates. In this study, serum binding was evaluated by a spectrophotometric method which measures the ability of BSA to compete with rat serum for binding bilirubin (19). Twenty-

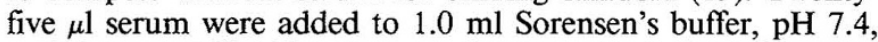
and the total bilirubin concentration was determined from the absorptivity at $450 \mathrm{~nm}$. Extinction coefficients for bilirubin bound to rat serum, rat/HSA serum, and BSA were determined at various wave lengths. Nonicteric rat serum had negligible absorptivity at the wave lengths measured. BSA has a higher molar absorptivity at longer wavelengths than either rat or human serum albumin, with the maximal difference occuring at $485 \mathrm{~nm}$. After determining the serum bilirubin concentration, the change in absorption at $485 \mathrm{~nm}$ was measured after adding $20 \mu \mathrm{l} \mathrm{BSA}$ solution $(3 \mathrm{~g} / \mathrm{dl})$ to the cuvette. The relative concentrations of BSA-bilirubin and rat serum bilirubin were then determined. In a model system, where bilirubin is distributed between primary binding sites of BSA and rat or human albumin, titration of icteric serum with BSA will theoretically yield a complete description of serum binding. In these experiments, where the bilirubin/albumin ratio exceeded 1.0, only semiquantitative data could be obtained. BSA served as a nonsaturable alternative receptor similar to Sephadex G-25. The binding index was defined as the percent total serum bilirubin transferred to BSA (100 $\times$ BSA-bilirubin/total serum bilirubin). High values of binding index reflect weak serum binding and a high free bilirubin concentration.

EEG changes following or during bilirubin infusion were classified as none (normal), a decrease in amplitude of $20-60 \%$ over one or both hemisphere (moderate) (Fig. $1 D^{\prime}$ ), or a decrease in amplitude of $80-100 \%$ over both hemispheres (severe) (Fig. $1 D$ ). In some animals, EEG changes progressed for 2-4 min after bilirubin infusion was stopped. The EEG was scored according to the lowest amplitude observed.

Stepwise discriminant analysis (20) was performed to analyze the extent to which total bilirubin concrentration, bilirubin binding index, and brain bilirubin concentration in each hemisphere contributed to the development of EEG abnormalities. The discriminatory procedure first selected that variable which was the best single discriminator of the three EEG groups. Additional variables were selected stepwise until the addition of another variable did not improve significantly the ability to discriminate between the three groups. Significance of differences in means in the three groups was assessed using the Newman-Keuls multiple range test for unequal sample sizes (21).

\section{RESULTS}

In five rats, we were unsuccessful in opening the BBB, and none of these animals developed staining or EEG changes. Of the remaining 22 animals (nine receiving HSA), seven maintained normal cortical electrical activity during and following the bilirubin infusion, five developed some decrease in amplitude over one or both hemispheres, and 10 developed flattening or near flattening of the EEG over both hemispheres. Typical 
responses are illustrated in Figure 1. In contrast to previous experience (17), infusion of HSA did not uniformly protect rats from EEG changes. However, the four HSA infused animals who developed moderate (3) or severe (1) encephalopathy had either poor serum binding despite the HSA infusion (more than $40 \%$ bilirubin bound to BSA) or deep staining of the right cerebral hemisphere.

Qualitative confirmation that the deeply stained right hemisphere was due to the movement of albumin-bilirubin across an open BBB was obtained by infusing Evans Blue dye intravenously 5 min after the infusion of bilirubin was terminated. Evans Blue binds to albumin without displacing bilirubin and will move into the interstitium if the barrier is permeable to proteins. When the $\mathrm{BBB}$ was opened, the dye produced a green color (bilirubin plus Evans Blue) in the right hemisphere and a small portion of the left frontal lobe which receives its blood supply from the common anterior cerebral artery. Separate blue or yellow stained areas were not observed. Staining was usually greatest in the temporal lobes and often extended into the thalamus and sometimes hypothalamic structures. The dorsum of the brain was variably stained.

The mean total bilirubin concentration was similar in all three groups (Table 1). The bilirubin content of the right hemisphere, reflecting the degree of BBB opening, was higher in rats with severe encephalopathy than in normal rats. Although the mean binding index appeared to be higher in the severely affected group, the difference did not reach statistical significance. There was no statistical correlation between bilirubin content in either right or left hemisphere and binding state. Most of the left hemisphere bilirubin content was presumed, therefore, to represent some cross-over effect of arabinose on the BBB and not movement of free bilirubin across an intact BBB.

Comparison of mean values (Table 1) can be deceptive when there is interaction between independent variables in producing an event. In this study, only the difference between "severe" and "normal" mean right hemispheric bilirubin content reached statistical significance. Possible interaction of bilirubin binding and right hemispheric barrier opening in the development of encephalopathy is suggested by Figure 2 which plots affected and nonaffected animals as a function of binding versus brain bilirubin concentration. When the free bilirubin was relatively low, i.e. when less bilirubin was transferred to BSA, encephalopathy occurred only when the barrier was very permeable to the albumin-bilirubin complex. When the serum binding was poor, encephalopathy developed at a much lower brain bilirubinalbumin concentration. Using a similar plot (Fig. 3), total bilirubin concentration did not appear to improve the predictive value of brain bilirubin alone.

Stepwise discriminant analysis confirmed that differences in

Table 1. Bilirubin values observed in relation to encephalopathy $( \pm S D)$

\begin{tabular}{lccc}
\hline \multicolumn{1}{c}{ EEG Change } & Normal & Moderate & Severe \\
\hline$n$ & 7 & 5 & 10 \\
Bilirubin $(\mathrm{mg} / \mathrm{dl})$ & 37.7 & 40.0 & 35.9 \\
& $( \pm 6.0)$ & $( \pm 5.7)$ & $( \pm 14.0)$ \\
Binding index $(\% \mathrm{ex}-$ & 24.8 & 25.6 & 51.5 \\
tracted) & $( \pm 21.8)$ & $( \pm 13.4)$ & $( \pm 28.8)$ \\
Right brain $(\mu \mathrm{g} / \mathrm{g})$ & 17.9 & 23.4 & $30.8^{*}$ \\
& $( \pm 6.4)$ & $( \pm 4.8)$ & $( \pm 9.8)$ \\
& 10.6 & 12.2 & 14.3 \\
Left brain $(\mu \mathrm{g} / \mathrm{g})$ & $( \pm 1.7)$ & $( \pm 4.6)$ & $( \pm 4.5)$ \\
\hline
\end{tabular}

* Severe versus normal $p<0.025$; remaining mean differences are not significant ( $p>0.05$, Neumann Keuls test).

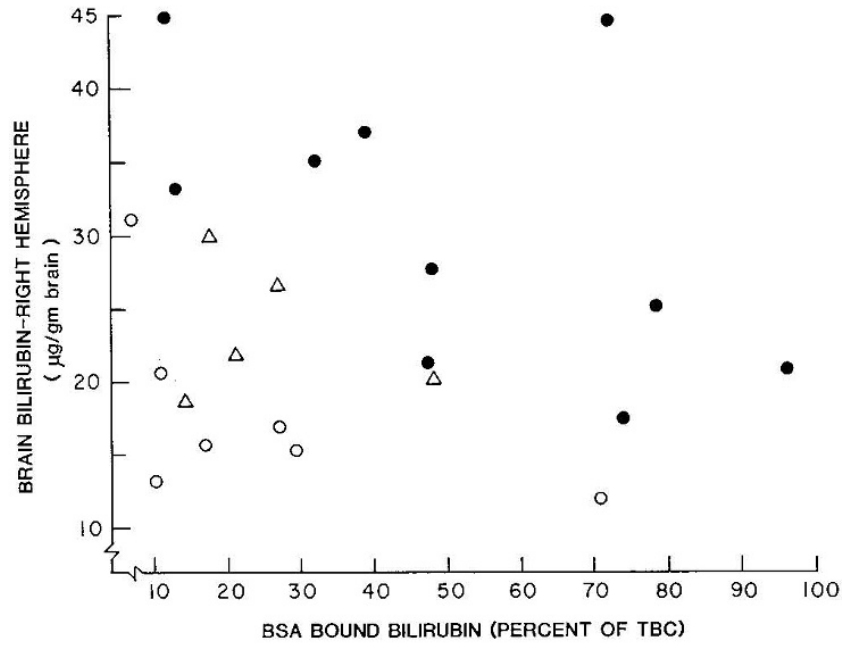

Fig. 2. Occurrence of EEG changes in relation to BBB opening in the right hemisphere and serum binding. With weaker binding (increasing free bilirubin) a higher percentage of total bilirubin is bound to BSA. EEG changes: $\bigcirc$ none, $\triangle$ moderate, $\bullet$ severe.

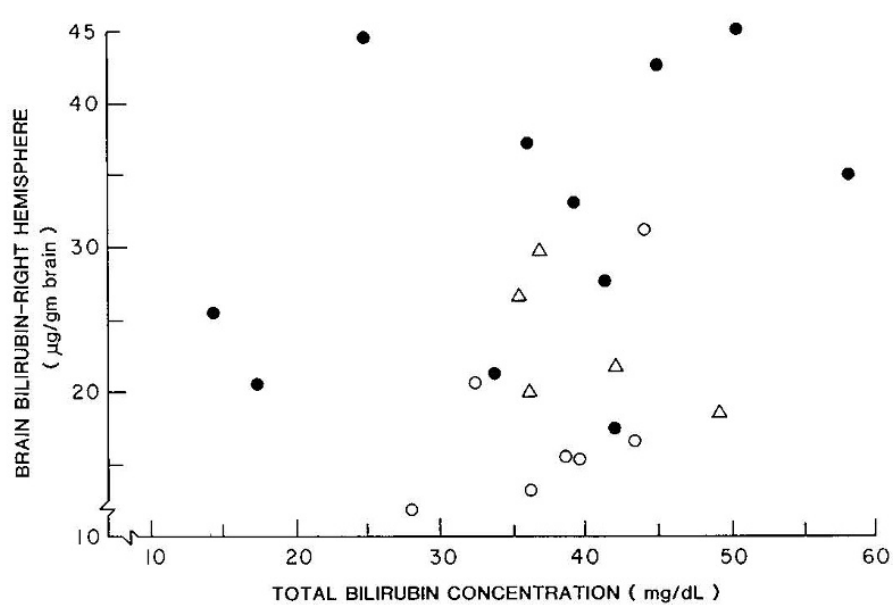

Fig. 3. Occurrence of EEG changes in relation to BBB opening in the right hemisphere and total bilirubin concentration. EEG changes: $O$ none, $\Delta$ moderate, $\bullet$ severe.

Table 2. Discriminant analysis: prediction of encephalopathy*

\begin{tabular}{lrccc}
\hline & & \multicolumn{3}{c}{ Predicted group membership } \\
\cline { 3 - 5 } Actual group & $n$ & Normal & Moderate & Severe \\
\hline Normal & 7 & 6 & 1 & 0 \\
Moderate & 5 & 1 & 4 & 0 \\
Severe & 10 & 0 & 2 & 8 \\
\hline
\end{tabular}

* Discriminant analysis: EEG was best predicted by two discriminators: binding index and right hemisphere bilirubin concentration.

EEG findings could best be discriminated by two variables, the concentration of bilirubin in the right hemisphere and binding index. Total bilirubin concentration and left hemispheric bilirubin were not useful discriminating variables. The two discriminating factors classified $18 / 22$ rats correctly, and in no case was a normal EEG misclassified as severe encephalopathy (Table 2).

\section{DISCUSSION}

We have confirmed that osmotic opening of the BBB produces yellow staining of the brain in icteric rats. However, yellow 
staining occurred in some animals without EEG evidence of bilirubin toxicity. The risk for encephalopathy depended on both the degree of barrier disruption as defined by brain bilirubin content and the serum binding status. In these short-term experiments we could not produce toxicity without first disrupting the BBB.

The concentration of bilirubin-albumin, i.e. total bilirubin concentration, did not discriminate which animals would develop encephalopathy. Thus, these data do not support the hypothesis that albumin bound bilirubin crossing the $\mathrm{BBB}$ is toxic to the brain (8). However, disruption of the BBB permits leakage of the albumin-bilirubin complex into the interstitium of brain, bathing the neuropil in icteric serum and exposing neurons directly to the same free bilirubin concentration existing in serum. Under these conditions albumin provides a large and relatively static reservoir of bilirubin which will equilibrate with alternative binding sites on albumin or cellular membranes. If the free bilirubin level is sufficiently high and the reservoir sufficiently large, the amount of bilirubin bound to membranes will impair neuronal function resulting in abnormal electrical activity.

This study was designed to establish principles regarding the relative importance of total bilirubin levels, binding, and $\mathrm{BBB}$ opening with respect to neurotoxicity and not to imitate the neurophysiological state of the newborn. One must be cautious, therefore, in making direct extrapolations from these animal experiments to human kernicterus. Although the basal ganglia were usually involved, the staining of brain in these animals was diffuse, involving cortical gray matter as well as deeper structures. These studies permit the possibility that variations of $\mathrm{BBB}$ permeability to albumin may explain variations in susceptibility of human newborns to bilirubin staining and toxicity, but they provide no direct evidence that the $\mathrm{BBB}$ is altered in infants who develop kernicterus.

Two additional factors that might be important in explaining the variability of the newborn's response to bilirubinemia were not addressed in this study, i.e. differences in the transport of free bilirubin across the BBB (22) and variations in cell response to a given bilirubin load. Variations in susceptibility of target neurons, e.g. due to chronic or acute ischemia, have been implicated in the pathogenesis of kernicterus. In this study, infusion of hypertonic arabinose produced a transient change in EEG indicating at least a transient alteration in brain function (23). It is possible, therefore, that disrupting the BBB or exposing neural tissue to arabinose altered brain metabolism $(24,25)$, increasing neuronal susceptibility to bilirubin.

This experimental model supports the proposal that yellow staining cannot be equated with bilirubin toxicity and provides a possible explanation for the neuropathological observations of Turkel et al. (26). In many sick premature infants with yellow staining, they found spongy changes in brain consistent with interstitial edema (or interstitial bilirubin-albumin), but few changes in neurons that could be attributed to bilirubin. The presence of yellow "staining" in some rat brains without evidence of encephalopathy reinforces the need to define bilirubin encephalopathy on the basis of behavioral (27), physiological (28), neurological (29), or histopathological criteria (30) and not on gross neuropathological findings.

Acknowledgments. The authors thank Drs. Jorgen Jacobsen, Albert Gedde, David Barry, and Bent Friis-Hansen for their advice and support during the early phase of these studies, and to Dr. Charles Franti for his advice regarding statistical analysis.

\section{REFERENCES}

1. Allen FH Jr, Diamond LK, Vaughan VC III 1950 Erythroblastosis fetalis: II. Prognosis in relation to history, maternal titer and length of gestation. Pediatrics 6:441-451

2. Hsia DYY, Allen FH, Gellis S, Diamond LK 1952 Erythroblastosis fetalis: VIII. Studies of serum bilirubin in relation to kernicterus. N Engl J Med 247:668-681

3. Cashore WJ, Oh W 1982 Unbound bilirubin and kernicterus in low-birth weight infants. Pediatrics 69:481-485

4. Brodersen R 1980 Binding of bilirubin to albumin. CRC Crit Rev Clin Lab Sci 11:305-399

5. Odell GB, Cohen SH, Kelly PC 1969 Studies in kernicterus. II. The determination of the saturation of serum albumin with bilirubin. J Pediatr 74:214 230

6. Johnson L, Boggs T 1974 Bilirubin-dependent brain damage: incidence and indications for treatment. In: Odell GE, Simopoulos AP, Shaff R (eds) Phototherapy in the Newborn: An Overview. National Academy of Sciences, Washington, DC, pp 122-149

7. Valaes T, Kaputulnik J, Kaufmann NA, Blondheim SH 1976 Experience with Sephadex gel filtration in assessing the risk of bilirubin encephalopathy in neonatal jaundice. Birth Defects 12:215-228

8. Levine RL, Fredericks WR, Rapoport SI 1982 Entry of bilirubin into the brain due to opening of the blood brain barrier. Pediatrics 69:255-259

9. Rapoport SI, Fredericks WR, Ohno K, Pettigrew KD 1980 Quantitative aspects of reversible osmotic opening of the blood-brain barrier. Am J Physiol 238:R421-431

10. Chiueh CC, Sun CL, Kopin IJ, Fredericks WR, Rapoport SI 1978 Entry of ${ }^{3} \mathrm{H}$-norepinephrine, ${ }^{125} \mathrm{I}$-albumin and Evans Blue from blood into brain following unilateral osmotic opening of the blood-brain barrier. Brain Res 145:291-301

11. Day R 1954 Inhibition of brain respiration by bilirubin: reversal of inhibition by various means. Proc Soc Exp Biol Med 85:261-264

12. Lie SO, Bratlid D 1970 The protective effect of albumin on bilirubin toxicity on human fibroblasts. Scand J Clin Lab Invest 26:37-41

3. Menken M, Waggoner JG, Berlin NI 1966 The influence of bilirubin on oxidative phosphorylation and related reactions in brain and liver mitochondria: effect of protein-binding. J Neurochem 13:1241-1248

14. Cowger ML 1971 Mechanism of bilirubin toxicity on tissue culture cells: factors that affect toxicity, reversibility by albumin, and comparisons with other respiratory poisons and surfactants. Biochem Med 5:1-16

15. Silberberg DH, Johnson L, Ritter L 1970 Factors influencing toxicity of bilirubin in cerebellar tissue culture. J Pediatr 77:386-396

16. Nelson T, Jacobsen J, Wennberg RP 1974 Effect of $\mathrm{pH}$ on the interaction of bilirubin with albumin and tissue culture cells. Pediatr Res 8:963-967

17. Wennberg RP, Hance AJ, Jacobsen J 1984 The importance of serum binding and the blood brain barrier in the development of acute bilirubin toxicity, In: Rubaltelli FF, Jori G (eds) Neonatal Jaundice: New Trends in Phototherapy. New York, Plenum Press, pp 35-43

18. Bratlid D, Winsnes A 1971 Determination of conjugated and unconjugated bilirubin methods based on direct spectrophotometry and chlorofrom extraction: a reappraisal. Scand J Clin Lab Invest 28:41-48

19. Gray RD, Stroupe SD 1978 Kinetics and mechanism of bilirubin binding to human serum albumin. J Biol Chem 253:4370-4377

20. Press SJ 1972 Applied Multivariate Analysis. Holt, Rinehart, and Winston, Inc., New York, pp 369-387

21. Zar JH 1974 Biostatistical Analysis. Prentice-Hall, Inc., Englewood Cliffs, NJ, pp 151-156

22. Pardridge WM 1981 Transport of protein-bound hormones into tissues in vivo. Endocrine Rev 2:103-123

23. Fieschi C, Lenzi GL, Zanette E, Orzi F, Passero S 1980 Effects on EEG of the osmotic opening of the blood-brain barrier in rats. Life Sci 2:239-243

24. Pappius HM, Savaki HE, Fieschi C, Rapoport SJ, Sokoloff L 1979 Osmotic opening of the blood-brain barrier and local cerebral glucose utilization. Ann Neurol 5:211-219

25. Rapoport S, London ED, Fredericks WR, Dow-Edwards DL, Mahone PR 1981 Altered cerebral glucose utilization following blood-brain barrier opening by hypertonicity or hypertension. Exp Neurol 74-519-529

26. Turkel SB, Miller CA, Guttenberg ME, Moynes DR, Hodgman JE 1982 A clinical pathologic reappraisal of kernicterus. Pediatrics 69:267-272

27. Golub H, Corwin MJ 1982 Infant cry: a clue to diagnosis. Pediatrics 69:197201

28. Wennberg RP, Ahlfors CE, Bickers RG, McMurtry CA, Shetter JL 1982 Abnormal auditory brainstem response in a newborn infant with hyperbilirubinemia: Improvement with exchange transfusion. J Pediatr 100:624-626

29. Van Praagh R 1961 Diagnosis of kernicterus in the neonatal period. Pediatrics 28:870-876

30. Ahdab-Barmada M, Moossy J 1984 The neuropathology of kernicterus in the premature neonate. J Neuropathol Exp Neurol 43:45-56 